\title{
EDUCATION
}

\section{Incorporating A Structured Writing Process into Existing CLS Curricula}

\author{
KAREN HONEYCUTT, SANDRA LATSHAW
}

\begin{abstract}
Good communication and critical thinking are essential skills for all successful professionals, including Clinical Laboratory Science/Medical Laboratory Science (CLS/MLS) practitioners. Professional programs can incorporate writing assignments into their curricula to improve student written communication and critical thinking skills. Clearly defined, scenario-focused writing assignments provide student practice in clearly articulating responses to proposed problems or situations, researching and utilizing informational resources, and applying and synthesizing relevant information. Assessment rubrics, structured feedback, and revision writing methodologies help guide students through the writing process. This article describes how a CLS Program in a public academic medical center, located in the central United States (US) serving five centrally-located US states has incorporated writing intensive assignments into an existing 11-month academic year using formal, informal and reflective writing to improve student written communication and critical thinking skills. Faculty members and employers of graduates assert that incorporating writing intensive requirements have better prepared students for their professional role to effectively communicate and think critically.
\end{abstract}

ABBREVIATIONS: APA - American Psychological Association, ASCLS - American Society for Clinical Laboratory Science, ASCP - American Society for Clinical Pathology, CLS - Clinical Laboratory Science, CLS/MT - Clinical Laboratory Science/Medical Technology, CLS Program A - CLS Program in a public academic medical center, located in the central United States (US) serving five centrally located US states, CLSI - Clinical Laboratory Standards Institute, CT - critical thinking, MLA - Modern Language Association, MLS - Medical Laboratory Science, NAACLS - National Accrediting Agency for Clinical Laboratory Sciences, US - United States, University B centrally located US public university, WI - writing intensive

INDEX TERMS: Clinical Laboratory Science, Communication, Critical Thinking, Curriculum, Evaluation, Rubric, Teaching, Writing, Writing Intensive, Writing Across the Curriculum

Clin Lab Sci 2014;27(4):194

Karen Honeycutt, M.Ed., MLS(ASCP) ${ }^{C M} S M^{C M}$, Clinical Laboratory Science Program, School of Allied Health Professions, University of Nebraska Medical Center, Omaha, NE

Sandra Latshaw, MA, MT(ASCP)SM, Clinical Laboratory Science Program, School of Allied Health Professions, University of Nebraska Medical Center, Lincoln, $N E$

Address for Correspondence: Karen Honeycutt, M.Ed., MLS(ASCP) ${ }^{C M} S M^{C M}$, Program Director, Clinical Laboratory Science Program, School of Allied Health Professions, University of Nebraska Medical Center, 984010 Nebraska Medical Center, Omaha, NE 68198-4010,402-559-9045, khoneycu@unmc.edu

\section{INTRODUCTION}

Is competent writing a required entry-level skill for CLS/MLS professionals? A report of The National Commission on Writing, including a survey of 120 American corporations, states that writing skills appear to be an attribute of high-skilled, high-wage professional work. ${ }^{1}$ The National Accrediting Agency for Clinical Laboratory Sciences (NAACLS) Standards for Clinical Laboratory Science/Medical Technology (CLS/MT) Programs state, "entry-level CLS/MT practitioners will possess basic knowledge, skills, and relevant experiences in communications to enable consultative interactions with members of the healthcare team, external relations, customer service and patient education." ${ }^{2}$ The ASCLS Body of Knowledge 


\section{EDUCATION}

lists communication skills as an essential function of a CLS communicating effectively in written and spoken English. ${ }^{3}$ Laboratory professionals may write policies, procedures, memoranda, guidelines, and quality assurance reports for peers and other health care professionals. ${ }^{4}$ Incorporating writing assignments into CLS curricula provides student practice to improve communication skills within the practitioner context.

Few would argue that good critical thinking skills (CT) are a desirable CLS professional trait. Paul and Sciven state, "critical thinking is the intellectually disciplined process of actively and skillfully conceptualizing, applying, analyzing, synthesizing, and/or evaluating information gathered from or generated by, observation, experience, reflection, reasoning or communication as a guide to belief and action." Kenimer's national survey of laboratory practitioners identified 65 CT behaviors specifically important in the CLS practice setting. ${ }^{6}$ Examples of these CLS professional specific CT behaviors that also correlate with Paul and Sciven's broad CT definition include: using work experience to make judgments and decisions about tasks, seeking and using relevant information and data to make appropriate decisions, clearly summarizing work questions or concerns, justifying work decisions, revising actions based on feedback of others, and communicating with coworkers.,

Kurfiss focuses the critical thinking definition around problem-solving as "critical thinking is an investigation whose purpose is to explore a situation, phenomenon, question or problem to arrive at a hypothesis or conclusion integrating all available information for justification." ${ }^{\text {P }}$ Problem-solving writing assignments help learners develop critical thinking skills by requiring students to comprehend a given question or situation, determine what information is needed to address the situation, determine where and how to gather relevant information, synthesize and apply the gathered information into a response, and clearly articulate the reasoning supporting the response.

Can writing promote critical thinking as well as communication skills? Writing is both a process of critical thinking and a product communicating critical thinking results. ${ }^{8,9}$ Writing focused on a scenario should reveal the writer's active engagement in communicating an organized response, supported by evidence-based reasoning. Employing critical thinking skills, writers work through a process of exploration resulting in drafts and revisions to improve reasoning and writing mechanic skills.

Should writing assignments be incorporated into science-intensive curricula? The National Commission on Writing ${ }^{1}$ recommends that educators double the amount of time students currently spend on writing and assign that writing across the curriculum. Writing intensive (WI) courses focus on assignments that allow students to write as a particular profession writes. For example, CLS students can focus on writing technical procedures or comparing two laboratory methods. Providing professional practice experience, where one learns to think and perform like a laboratory professional, is the CLS educational signature pedagogy. ${ }^{10}$ Requiring CLS specific problem-solving writing assignments allows students to practice and develop CLS specific CT behaviors. ${ }^{6}$

\section{BACKGROUND}

The CLS Program at this centrally located, public academic medical center (CLS Program A) is an 11month, $3+1$ program with approximately 60 students in five centrally-located states at 17 clinical sites. Clinical Laboratory Science Program A has contractual relationships with five other centrally located US public and private universities, one of which will be referred to as University B. CLS Program A is responsible for providing all CLS educational components. Responsibility for student services and clinical sites lies with each individual contract university. Students from these universities can earn their CLS baccalaureate degree from the contract university and/or a certificate of completion from the NAACLS approved CLS Program A.

The impetus for incorporating writing intensive courses into existing curricula was initiated due to the relationship with University B. Each University B undergraduate is required to successfully complete two WI courses with one course in their primary area of study. Finding time for students to complete additional work in the already overwhelming program did not seem feasible, but was a necessity. Due to deadline constraints, CLS Program A faculty developed and implemented two University B approved WI courses over a four-month period. The WI assignments are 


\section{EDUCATION}

concurrently scheduled with clinical rotations. The benefits of expanding writing expectations proved valuable to all students in the CLS Program.

\section{WRITING INTENSIVE STRATEGIES}

The WI courses are approved yearly by the University B Campus Writing Program. ${ }^{11}$ Qualified WI instructors should be professionals that write in their discipline as a practitioner, not necessarily grammar experts. Writing assignments tailored to a program's profession must total a minimum of 20 pages, including at least eight revision pages, and be a major component of a WI course grade. Assignments revolve around problems within the profession's scope of practice requiring writers to identify and challenge assumptions, consider opposing views, justify resolutions, and communicate in a manner appropriate to that profession.

Explicit WI directions should include the assignment purpose, student expectations, deadlines, assessment forms and grading criteria. The writer's role related to the scenario must be explained. Is the student writing from the perspective of a CLS practitioner, manager, or student? Is the writer's audience a pathologist, laboratory manager or professional peer? Directions should specify the resources students can use to address the scenario. Some assignments require students to find evidence-based resources using library databases. Other assignments direct students to professional websites or provided articles. Is the major learning objective finding the evidence or is it assimilating and applying that evidence or are both equally important? This may depend on the writer's prior knowledge and skill-level in seeking and interpreting appropriate reference materials.

Assignment directions describe the final writing format (e.g., laboratory procedure, report). Templates, such as a laboratory procedure, can focus the content for each required component. What technical style (e.g., APA, MLA) and version should be used? To resolve inconsistencies, standard writing guidelines for all student assignments were developed. Quality examples of an assignment can demonstrate the expected caliber of student work.

Specific feedback helps the writer improve communication skills. Facilitative remarks provide explicit, guiding feedback assisting students in improving their argument justification. Evaluators should avoid vague feedback such as 'explain in more detail'. Provide feedback with leading, probing questions:

- How does the test improve patient outcomes?

- What level of expertise is needed to perform both test methods?

Directive, 'explicit' remarks focus on inaccurate or incomplete concept discussion:

- Provide specific sensitivity and specificity data.

- What is the purpose of 'Reagent $A$ ' in the test reaction?

Writing mechanics are not corrected, but rather comments such as 'grammar errors' or 'confusing word order' are provided so writers learn how to correct their own errors.

Summary comments provided at the end of an assignment address the writer's overall adherence to the assignment. The evaluator notes strong and weak areas of the paper. Writing mechanics are not the primary focus unless problems are so prevalent that they distract from the writing's meaning and communication. Feedback should provide the writer with a sense of constructive guidance.

Writing assignments can be viewed on a continuum of informal to formal writing. Informal writing is "writing to learn," a low-stakes assignment that may or may not be graded. Examples include brainstorming ideas on paper, journaling, or early written drafts. Writing mechanics are not emphasized, as the primary audience is oneself or a peer. Formal writing is viewed as "writing to communicate," high-stakes, and a significant part of a student's grade. Examples include analytical or critical papers with a developed thinking process. Writing mechanics are of greater importance. The target audience is public or external, making clear communication essential. The writing assignments increase in complexity during the first semester throughout the revision process. The second semester writing goal is to expose students to a breadth of topics and writing styles.

To nurture and develop writing skills, formal papers may evolve through one or several revision steps. Research paper assignments may overwhelm a student 
with fledgling writing skills. Breaking an assignment into stages allows a student to focus on one or two primary tasks at each juncture. Feedback allows students to fine-tune their thinking before advancing to the next task. Specific comments guide the student's critical thinking process, leading them where they may not have ventured and steering them back on course when needed. Revision projects extend from several weeks to an entire semester.

The revision process may be a new concept to many students. Writing is a process that requires clear understanding of the issue, modification of initial ideas, rethinking of the argument, and communication of that argument. Revision is not merely editing writing mechanics.

\section{INFORMAL WRITING EXAMPLES Online Threaded Discussions}

Online threaded discussion is used to encourage communication between students at different clinical sites. Assignments focus on topics with differing viewpoints, providing students opportunities to learn from peers. A cultural competency assignment:

A non-English speaking patient comes to the laboratory to have blood drawn. The interpretive services coordinator is not available. The phlebotomist is in the ER. You are now responsible for collecting the specimen from this patient. What communication protocol will you use with this patient?

Students, assigned to groups of four, conduct threaded discussions on approaches for assessing the patient's language and securing appropriate interpretive services at the student's clinical facility.

Each group member posts an original response and replies to at least two other student threads, by adding to their comments. Postings should apply cultural competency course information. Each group submits a final summary. Threaded discussions are graded to encourage student participation using a short assessment with two points possible for each of five rubrics assessing an individual's initial posting, responses to team member postings, timeliness, and quality of the group summary. The emphasis is on the content and quality of discussion, not writing mechanics.

\section{Remediation work}

All failed exams at CLS Program A require remediation. Students critically think through missed questions and communicate their thought process in writing by providing the correct answer, thoroughly explained with references, and an explanation for all distracters. A student must use sufficient writing mechanics to make their intent clearly understood, but the emphasis remains on bolstering content comprehension. Remediation is reviewed, but has no effect on the student's original grade.

\section{FORMAL WRITING ASSIGNMENT EXAMPLES, NON-REVISED}

\section{Opinion or Persuasive Papers}

Opinion or persuasive papers tackle issues that have two opposing positions. Students are required to investigate personnel licensure for laboratory professionals and write an article for a laboratory's newsletter to include:

- Differentiation of licensure from certification

- Key pro and con arguments

- ASCLS and ASCP positions

- Potential effects on patient care, the community, health care costs, vacancy rates, physicians, pathologists, MLS/MLT, other healthcare workers

- Justification of the position the student chooses to support

- Opinion papers receive a score of 0-4, using a holistic assessment (Table 1) with expectations defined for each score. Grades are dependent on how well students focus on the assigned task, the overall organization and development of the topic, and the format and writing mechanics of their paper. The focus remains on content accuracy and the student's clearly communicated position justification. Poor writing mechanics can lower a student's grade by a maximum of one point.

\section{Reflective papers}

Completing professional activities is a program requirement. Students earn 7-10 professionalism points in the categories of professional development, community service and scholarly activity. ${ }^{12}$ Student contemplation on these experiences is as valuable as their participation in the activities. Near graduation, students write a personal reflection of their individual 
Table 1. Holistic Grading Assessment.

4 = Paper is very good in content, organization, development, mechanics and format

The paper focuses on the assigned task, accurately and thoroughly addressing all questions implicit in the assignment. The content engages the reader, shows author insights and is written at an appropriate professional level. Organization is logical and easily followed; paragraphs are developed with pertinent examples or citations. There are rare, if any, distracting errors in grammar, spelling, or sentence structure. Format follows 'CLS Writing Guidelines for Students' with minor exceptions.

\section{3 = Paper is competent in content, organization, development, mechanics and format}

The paper focuses on the assigned task, accurately and thoroughly addressing most questions implicit in the assignment. The content usually does not engage the reader, focusing primarily on obvious responses that most writers are likely to provide and/or the writing is not always at a professional level. Organization is generally logical, but may be a bit difficult to follow in places because the writer has omitted transitions or connections or failed to develop paragraphs with adequate or appropriate examples and citations. Format generally follows 'CLS Writing Guidelines for Students'.

\section{2 = Paper is inadequate in content, organization, development, mechanics and format}

The paper fails to focus on the assigned task and/or fails to accurately and thoroughly answer the questions implicit in the assignment. The content usually does not engage the reader, focusing primarily on obvious responses that most writers are likely to provide and/or the writing is not at a professional level. Organization may be logical, but transitions or connections are often omitted and/or paragraphs are inadequately developed throughout. Format inconsistently follows 'CLS Writing Guidelines for Students'.

$\mathbf{1}=$ Paper is weak in content, organization, development, mechanics and format

The paper fails to focus on the assigned task and fails to accurately and thoroughly answer the questions implicit in the assignment. The content is not engaging and is superficial; the writing is not professional. Organization may be logical, but transitions and connections are usually omitted and paragraphs are thinly developed. Format rarely follows 'CLS Writing Guidelines for Students'.

\section{Unacceptable/Student must redo paper}

The paper is unacceptable in content, organization, development, and style. The paper fails to focus on the assigned task. The content is exceedingly superficial; the writing is not professional. The organization is random and confusing and paragraphs are undeveloped. Format does not follow 'CLS Writing Guidelines for Students'.

\section{All items listed under each level must be met for the student to achieve that grading level. A half point (0.5) may be added to a student's level if they meet the majority of criteria of the next higher level.}

\section{Writing Mechanics}

For errors in grammar, spelling and sentence structure, the following points are to be deducted from the above earned score: 0.5 points deducted for $3-5$ errors noted in the paper

1 point deducted for 6 or more errors noted in the paper

professional activities assessing how or if these experiences will influence their future professional practice. The assignment is graded using the holistic assessment (Table 1).

\section{Writing a Laboratory Procedure}

Writing a laboratory procedure using Clinical Laboratory Standards Institute (CLSI) guidelines is a prescriptive assignment that follows a recommended format. Students are provided the same test package insert, CLSI guidelines and a template to construct their procedure. Clear communication and excellent writing mechanics are essential in laboratory procedures, so this assessment (Table 2) consists of rubrics for both. The content assessment (75\%) lists all criteria addressed in the template. Each criterion is assessed using standard $0-4$ quality indicators. Writing elements (paragraph structure and transitions, format, writing mechanics: grammar/spelling/sentence structure, and writing to audience) constitute $25 \%$ of the assessment. These rubrics describe unique $0-4$ quality indicators for each writing element.

\section{FORMAL WRITING ASSIGNMENTS REVISED Method Evaluation paper}

Students analyze provided comparison study data to statistically evaluate two generic methods leading to a selection justification. A condensed version of the student directions: 
Table 2. Excerpts* from the Writing a Laboratory Procedure Assessment.

\title{
Assessment of Content 75\%:
}

$4=$ Very good, above average: all or most required information is included with only minor omissions; all statements are accurate.

$3=$ Good, average: most required information is included with a few major omissions; most statements are accurate.

$2=$ Fair, below average: some required information is included but there are several major omissions and/or several statements are inaccurate.

$1=$ Poor: minimal required information is included.

$0=$ Unacceptable: rubric content was skipped; none of the required information is included

\section{Heading/Procedure Title/Page Format:}

_ Appropriate demographics, author, date, procedure being replaced, title, page numbers

\section{Principle:}

- Type of method used, specific substance measured and reaction sequence

- How response is measured and how measured response is related to final test result

\section{Clinical Significance:}

- Medical usefulness and clinical rationale of measured substance

_ Causes of increased/decreased test results

\author{
Specimen: \\ _ Patient preparation, sample collection criteria, specimen type and amount \\ - Specimen stability and specific handling and storage requirements \\ - Criteria for unacceptable specimens and action to follow when unacceptable specimen is received
}

\section{Assessment of Writing (25\%):}

\section{Paragraph structure and transitions}

$4=$ Paragraphs are coherent and unified with all sentences focusing on a single topic; logical transitions between paragraphs are made

$3=$ Paragraphs are generally coherent, unified and focused, but sometimes change direction midstream or leave minor gaps in the thought flow; paragraph transition is usually logical; 2 errors noted

$2=$ Paragraphs may be incoherent, have several gaps in the thought flow and/or address a mix of topics; paragraph transitions are often illogical and difficult to follow; 3 errors noted

$1=$ Paragraphs are often incoherent, have frequent gaps in the thought flow and address a mix of topics; paragraph transitions are illogical and difficult to follow; 4 errors noted

$0=$ Paragraphs are incoherent, have numerous gaps in the thought flow and address a mix of topics; paragraph transitions are poor making it difficult to follow; $>4$ errors noted

${ }^{*}=$ shows a selected 8 of 24 content-based rubrics and 1 of 4 writing-based rubrics from the complete assessment

You have been working on a project to compare a new insulin method to the insulin method currently in use at your laboratory. You have completed the data collection and begun statistical analyses when notified that you will be deployed. Your supervisor asks you to compile your results into a narrative paper detailing your findings for your successor. Your paper needs to include explanations and interpretations for all comparison studies performed.

Writing this paper proves difficult for many students, as they have trouble with the required critical thinking skills. The revision process helps develop students' skills and confidence. The first submission, worth $36 \%$ of the assignment, is assessed solely on content using a prescriptive assessment (Table 3) with standard 0-4 quality indicators. General comments are provided regarding writing mechanics that need improvement The final method evaluation paper submission is graded for content $(60 \%)$ with the same rubrics used for the initial paper. Writing quality (40\%) is graded using the writing rubrics previously described, with the addition of revision and organization categories.

\section{Research Paper}

Students compile detailed information on a new immunologic or molecular test method by applying research methods previously taught. The assignment 
Table 3. Excerpts* from the Method Evaluation Paper Final Assessment.

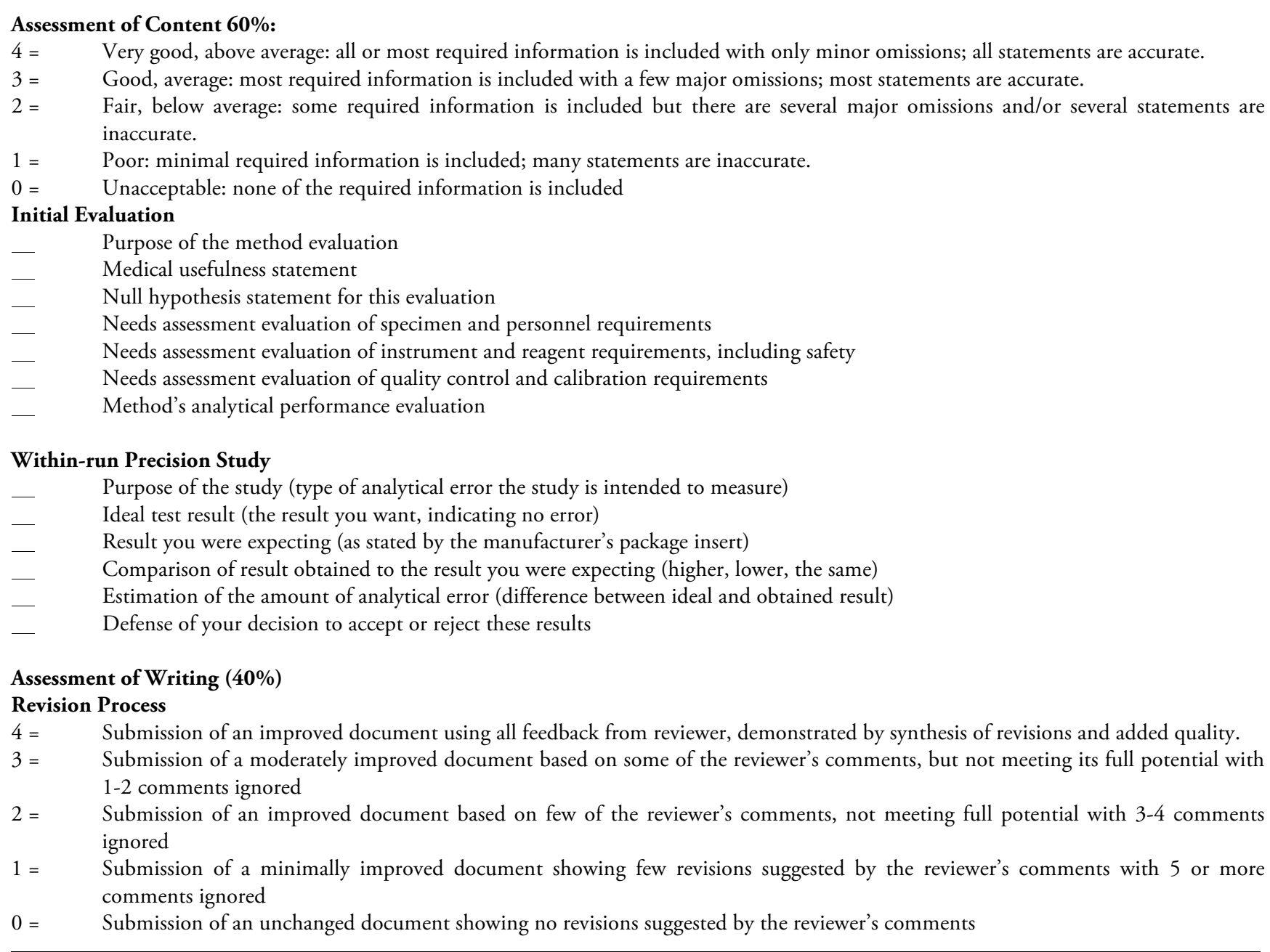

* $=$ shows a selected 13 of 45 content-based rubrics and 1 of 6 writing-based rubrics from the complete assessment

extends throughout a semester, with five written phases and a student-faculty conference. Once students determine a potential topic with sufficient relevant literature, they develop a prospectus, or preliminary examination of the topic's current research. This leads to an evidence-based thesis statement. Students provide at least six annotated references. Specific questions in the prospectus template lead students to consider all key factors.

Each rubric on the prospectus assessment form (Table 4) is scored 0-2 with two indicating that the needed information is thoroughly and accurately addressed; one if it is mentioned, but not fully or accurately developed; and zero if it has been overlooked. This allows for rapid grading, while providing students a checklist to assure required criteria are addressed. Following the grading of the prospectus, a student-faculty conference addresses concerns or problems. The second written stage is a revised prospectus. The same prospectus assessment is used, with the addition of a rubric regarding the quality of revision.

Submission of an introductory paragraph and paper outline comprise the third writing phase and is also graded with a $0-2$ point assessment. Students may need to repeat this step if the content quality is low. The first three stages are all low-stakes, with each step accounting for 10 percent of the assignment grade.

Adequate time must be allowed for students to compose and revise their writing as they prepare the entire rest of 
Table 4. Excerpts* from Research Paper Assessments.

Prospectus: The prospectus should display a clearly focused topic for the Immunology/Molecular Diagnostics research paper that has been thoroughly investigated prior to developing a thesis statement (a one sentence summary of your paper's focus.) A literature search focusing on the current and proposed immunology or molecular methods, their significance to the clinical laboratory and valid factors to be compared must be completed before anticipating possible outcomes and creating the thesis statement. The annotated bibliography provides a brief descriptive and evaluative paragraph reviewing the relevance, accuracy and quality of each source to be cited

The student will receive 0-2 points for each rubric criteria listed below (unless otherwise noted):

2 = rubric thoroughly addressed

$1=$ rubric incompletely or inaccurately addressed

$0=$ rubric not addressed

The library search was thoroughly investigated with a logical pathway

Both current and proposed new methods meet the assignment guidelines

The significance of this comparison for the laboratory and healthcare is discussed

An adequate amount of comparison factors are planned for discussion

Anticipated outcomes supported by comparison factors supported by annotated resources

The thesis statement is clearly written in one sentence addressing the paper's focus, two methods to be compared, comparison factors and the proposed outcome

The bibliography provides the required quantity of appropriate types of resources.

6 or more resources $=2 \mathrm{pts} ; 3-5$ resources $=1 \mathrm{pt} ; 0-2$ resources $=0$

The bibliography provides relevant references to support the thesis statement.

$5-6$ relevant sources $=2 \mathrm{pts} ; \quad 3-4$ relevant sources $=1 \mathrm{pt} ; \quad 0-2$ relevant sources $=0$

Annotations are a concise summary of an article's key content and relevancy to the topic.

5-6 quality annotations $=2$ pts; $3-4$ quality annotations $=1 \mathrm{pt} ; 0-2$ annotations $=0$

Writing is at an appropriate level to clearly describe the research focus.

First and Final Research Paper Assessment Rubrics for Content

Summary of laboratory applications

$4=\quad$ All key information (e.g., sensitivity, specificity, turnaround time, specimen requirements) about current and new laboratory applications of the selected testing is correctly summarized

$3=\quad$ Most key information about current and new laboratory applications of the selected testing is correctly summarized with 2-4 errors

$2=\quad$ Some information about current and/or new laboratory applications of the selected testing is summarized with 5-7 errors

$1=\quad$ Insufficient or incorrect information about the laboratory applications of the selected testing is summarized with 8-10 errors

$0=\quad$ Numerous errors and insufficient information about the laboratory applications of the selected testing is summarized with $>10$ errors

${ }^{*}=$ shows complete prospectus assessment and a selected 1 of 9 content-based first and final research paper rubrics from the complete assessment

the paper. Writing skills are not evaluated at this fourth stage, allowing a student to focus on content. The first paper assessment (30\% of assignment grade) describes unique 0-4 quality indicators for each criterion (Table 4).

The assignment culminates with the submission of a final research paper ( $40 \%$ of assignment grade). Most students earn high scores having utilized feedback to develop their writing skills gradually throughout the revision process. The content assessment rubrics are identical to those for the first submission research paper (Table 4). Writing skills are assessed using the writing rubrics previously discussed.

Examples of well-written research papers, in various stages, are provided for student and grader review. Due to the length of this paper and student numbers, multiple instructors are required to mentor students through the writing process. Standardized, clearly defined rubrics are essential for grading consistency.

\section{THE TOP TEN LESSONS LEARNED}

Five years of administering two WI courses has led to numerous insights, evolutions in curricula, and lessons learned.

1. Writing takes time! Multiple work sessions are often needed to develop critical thinking. Students are released from clinical work three hours each week to work on these assignments. Two dedicated weeks are allotted for larger 
writing assignments. Assignment distribution and deadlines must take into account other course demands.

2. Grading writing takes time! With approximately 60 students in WI courses, graders can easily be overwhelmed. Multiple instructors are designated for most writing assignments to distribute workload. One instructor grades all stages of revision assignments for consistency.

3. Feedback to students must be timely! Faculty may underestimate the time required to grade large papers, delaying student feedback and disrupting continuity of the revision process. Desired faculty turn-around-times are 48 hours for shorter papers, and one week for longer papers. Faculty to student ratios are closely examined and reassigned for each writing assignment to make these grading goals realistic and attainable.

4. Grading workshops encourage grading consistency. With multiple instructors grading the same assignment, personal interpretation of rubrics can be problematic. All graders are provided the same sample paper to individually grade with the existing assessment. Group discussion on each grader's scoring of assessment criteria leads to consensus on how rubrics will be interpreted.

5. Assignment instructions and assessments change from semester to semester! Feedback from faculty and students necessitate continual updating of writing assignment components to assure clarity for students and consistency in grading.

6. Assessment rubrics must be individually established for each assignment. One standard assessment does not fit all situations. However, the rubrics assessing writing skills should remain consistent for all assignments.

7. Count each writing mechanic error, such as the same spelling error, only once to avoid over penalizing a student for the same mistake.

8. Students must be required to gather needed information before any written assignments are submitted. Prior to establishing the research paper prospectus, many students selected inappropriate topics with insufficient supporting literature. Faculty assumed students would follow instructions to complete pre-requisite factfinding; however, student submissions proved differently. Forcing students to complete a prospectus as the initial assignment has eliminated rejected research topics.

9. Listen to student feedback! Regular evaluations of both individual writing assignments and entire courses are necessary. Students in early WI courses expressed there was too much writing, so assignments were reassessed for value. Four-point papers were misunderstood as not worth students' time, indicating instructions needed rewording to clarify these assignments are a percentage of the final grade, not just four points.

10. Additional resources may be needed for students with extremely poor writing skills. Some students require more guidance in basic writing than CLS instructors are equipped for or have time to provide. Formal writing centers are ideal for these situations, but most hospitals or professional campuses do not have these resources. Struggling students may be temporarily removed from writing courses, allowing them to focus on their clinical studies. Writing courses are completed at the end of an extended educational year, when students have fewer distractions and can take additional time to critically process writing assignments and hone their writing skills.

\section{STAKEHOLDER COMMENTS}

Faculty member regarding writing outcomes:

I have been associated with this CLS program for the last seven years and have graded writing assignments prior to the implementation of the writing intensive curriculum as well as following implementation. I feel that the quality of writing has improved significantly since the writing intensive curriculum was incorporated. The revision process allows the student to take feedback from the instructor and improve on their original submission. With every student I have worked with the quality of their writing improves throughout their clinical year.

Laboratory employer regarding importance of writing in the CLS profession:

Technologists are often asked for their input and ideas. Analytical thinking skills as well as the ability to convey these thoughts in a logical and straightforward manner can set those ideas above the ideas of others. A lack of basic 
communication skills continues to be one of the areas I find most lacking in professionals. Distinguishing appropriate modes of communication as well as presenting a grammatically correct and understandable message has long been a problem. Poor communication can result in:

- Misunderstanding and a lack of clarity leading to inefficiency

- Perceived lack of professionalism

- Perceived lack of knowledge

- Compromised patient safety

- Personal, institutional, and professional embarrassment.

For example, procedure writing, with its requirement for clear, factual, exact information presents a particular need that could easily create confusion and safety issues without solid writing skills.

Recent CLS graduate regarding relevance of a writing assignment:

Many people at the 2011 National ASCLS Conference were pleased to hear that our program required us to write a paper addressing the differences between certification, licensure and professional society membership, so kudos to the CLS program. The more I hear about different programs, the more I realize I was lucky to go through the one I did.

\section{DISCUSSION}

Literature supports the link between improved critical thinking through problem-focused writing. Exposing CLS students to various types of writing prepares them to communicate as a critically-thinking practitioner within the health care team. Faculty members of the CLS Program, as well as employers of the program's graduates, assert that incorporating writing intensive requirements has better prepared students for these professional roles. Proposed future WI outcome studies would objectively evaluate the quality of student critical thinking, communication and writing skills pre-and post-clinical year education.

\section{REFERENCES}

1. National Commission on Writing the College Board Advocacy Center [Internet]. New York: The Board; c2010 [updated 2004; cited 2011 Mar 28]. Writing a ticket to work or a ticket out: a survey of business leaders, [1-44]. Available from: http://www.host-collegeboard.com/advocacy/writing/publicati ons.html.

2. National Accrediting Agency for Clinical Laboratory Sciences [Internet]. Rosemont (IL): The Agency; c2008 [updated 2010 Oct 18; cited 2011 Mar 28]. Unique standards and documents section III required for accredited CLS/MT programs; [2-3]. Available from: http://www.naacls.org/accreditation/cls-mt/ docs.asp.

3. Anderson SC, editor. Body of knowledge clinical laboratory science content outline [CD-ROM]. Bethesda: ASCLS; 2004.

4. Warner S. Write for success: learn how to use words efficiently to convey your point for a host of lab documents. Advance for Medical Laboratory Professionals [Internet]. 2010 May [cited 2011 Mar 29];22(9):[about 2 p.]. Available from: http:// laboratorian.advanceweb.com/Features/Articles/Write-for-Suc cess-2.aspx.

5. Scriven M, Paul R. Defining critical thinking [Internet]. Tomales (CA): Foundation for Critical Thinking; 2009 [cited 2011 Mar 29]. Available from: http://www.criticalthinking. org/aboutCT/define_critical_thinking.cfm.

6. Kenimer EA. The identification and description of critical thinking behaviors in the practice of clinical laboratory science, part 1: design, implementation, evaluation, and results of a national survey. J Allied Health. 2002;31(2):56-63.

7. Kurfiss JG. Critical thinking: theory, research, practice and possibilities. ERIC Higher Edu Report No. 2, Washington (DC); 1988.

8. Bean JC. Engaging ideas the professor's guide to integrating writing, critical thinking, and active learning in the classroom. San Francisco: Jossey-Bass; 2001.

9. Alfaro-LeFevre R. Critical thinking in nursing, a practical approach. Philadelphia: W. B. Sanders Co; 1995.

10. Woeste LA, Barham BJ. The signature pedagogy of clinical laboratory science education: the professional practice experience. Lab Medicine. 2006;37(10):591-2.

11. University of Missouri-Columbia Campus Writing Program [Internet]. Columbia (MO): The Program; c2004 [updated 2007 Aug 13; cited 2011 Mar 30] Guidelines for writing intensive courses. Available from: http://cwp.missouri.edu/ wiforms/guidelines.htm.

12. Latshaw S, Honeycutt K. Professionalism - a required CLS/CLT curricular component. Clin Lab Sci. 2010;23(3) Suppl:24-31. 